\title{
Steroid Refractory Graft Versus Host Disease
}

National Cancer Institute

\section{Source}

National Cancer Institute. Steroid Refractory Graft Versus Host Disease. NCI Thesaurus.

Code C147114.

Graft versus host disease that does not respond to steroid treatment. 